\title{
PERFORMANCE OF CARBANP AND CIM TESTS IN OXA-48 CARBAPENEMASE-PRODUCING ENTEROBACTERIACEAE
}

\author{
SERAP SÜZÜK YILDIZ ${ }^{1 *}$, BANU KAŞKATEPE ${ }^{2}$, HAVVA AVCIKÜÇÜK ${ }^{3}$ and \\ ŞÜKRAN ÖZTÜRK ${ }^{2}$ \\ ${ }^{1}$ National Antimicrobial Resistance Laboratory, Public Health Institution of Turkey, \\ Ankara, Turkey \\ ${ }^{2}$ Department of Pharmaceutical Microbiology, Faculty of Pharmacy, Ankara University, \\ Ankara, Turkey \\ ${ }^{3}$ Clinical Microbiology Laboratory, 29 Mayıs Statement Hospital, Ankara, Turkey
}

(Received: 2 January 2017; accepted: 24 February 2017)

This study applied two phenotypic tests, namely "Carbapenemase Nordmann-Poirel" (CarbaNP) test and "Carbapenem Inactivation Method" (CIM), against the isolates carrying the carbapenem resistance genes. The study included 83 carbapenem-resistant Enterobacteriaceae isolates producing oxacillinase-48 (OXA-48) and 30 carbapenem-sensitive Enterobacteriaceae isolates. Out of the total isolates studied, 77 isolates $(92.77 \%)$ were identified as Klebsiella pneumoniae and six isolates (7.23\%) were identified as Escherichia coli by Matrix Assisted Laser Desorption Ionization-Time of Flight Mass Spectrometry. Polymerase chain reaction (PCR) method used to detect resistance genes found that 74 isolates $(89.16 \%)$ produced OXA-48 carbapenemase, whereas nine isolates $(10.84 \%)$ produced both OXA-48 and New Delhi metallo-beta-lactamase-1 (NDM-1). The isolates producing both OXA-48 and NDM-1 were found to be positive by both phenotypic tests. Among isolates carrying only $b l a_{\text {OXA-48 }}$ gene alone, nine isolates (13.04\%) for CarbaNP test and two isolates for CIM test $(2.90 \%)$ displayed false negative results, respectively. The sensitivity of CarbaNP and CIM tests was found to be $89.16 \%$ and $97.59 \%$, respectively, whereas the specificity was determined to be $100 \%$ for both tests. These findings suggest that CarbaNP and CIM tests are useful tools to identify the carbapenemase producers. Molecular methods like PCR are recommended to verify false negative tests predicted to have OXA-48 activity.

Keywords: carbapenem-resistant Enterobacteriaceae, carbapenemase, OXA-48, CarbaNP, CIM test

*Corresponding author; E-mail: serapsuzuk@gmail.com 


\section{Introduction}

The species included in the Enterobacteriaceae family are known to cause community-acquired and nosocomial infections. Carbapenems have been commonly used in the treatment of infections caused by these bacteria, which possess extended-spectrum beta-lactamases conferring resistance to many beta-lactam antibiotics [1]. Carbapenemase-producing Enterobacteriaceae (CPE) result in serious infections leading to an extension of the period of hospitalization and increase in the mortality ratio. Therefore, monitoring of development of resistance against carbapenems is necessary [2]. Carbapenemases contribute significantly to the development of resistance by hydrolysing the beta-lactam antibiotics [3]. These enzymes include class A carbapenemases like Klebsiella pneumoniae carbapenemase (KPC), class B carbapenemases like Verona integronencoded metallo-beta-lactamase, New Delhi metallo-beta-lactamase (NDM-1), imipenemase, and class D oxacillinases like OXA-48. Among them, OXA48-producing Enterobacteriaceae exist in Turkey endemically; metallobeta-lactamases like NDM-1 have also started to be encountered recently [4].

Although the molecular methods are considered to be the golden standard for the detection of carbapenemase encoding genes, these are not adaptable to all laboratories because of high cost and inaccuracy in the determination of new resistance genes. Therefore, the application of economical and rapid diagnostic screen tests has increased [5]. Rapid diagnosis of carbapenem-resistant isolates is necessary for taking appropriate precautions against infection during the early period and detection of the treatment procedure [6]. The most commonly used test is the "Carbapenemase Nordmann-Poirel (CarbaNP)" test, which is reported to have 100\% sensitivity and specificity. The test is based on the hydrolysis of the beta-lactam ring of a carbapenem, usually imipenem by bacterial lysate, which is determined by a $\mathrm{pH}$ indicator [7, 8]. Another phenotypic test that is cost-effective with $100 \%$ sensitivity and specificity is the "Carbapenem Inactivation Method (CIM)." It has been reported to be an effective phenotypic test for the identification of carbapenemase producers [9]. This study aimed to evaluate the performance of CarbaNP and CIM tests in the isolates carrying the $b l a_{\mathrm{OXA}-48}$ carbapenem resistance gene.

\section{Materials and Methods}

\section{Detection of isolates}

A total of 83 isolates obtained from the blood, urine, and tracheal aspirate were sent to 29 Mayis State Hospital, Microbiology Laboratory, Ankara, Turkey 
between 1 August 2015 and 30 November 2016. In addition, 30 Enterobacteriaceae isolates with no reduced sensitivity against any carbapenem were evaluated in this study. The isolates were identified using Matrix Assisted Laser Desorption Ionization-Time of Flight Mass Spectrometry (Bruker Biotyper; Bruker Daltonics, Bremen, Germany). The sensitivity of carbapenems, namely imipenem, meropenem, and ertapenem, was studied using the gradient strip test (Liofilchem, Roseto degli Abruzzi, Italy). The results of the antibiotic susceptibility test were evaluated according to EUCAST 2016 data [10].

\section{Detection of carbapenem resistance genes}

The existence of $b l a_{\mathrm{OXA}-48}$ and $b l a_{\mathrm{NDM}-1}$ genes was detected with the polymerase chain reaction (PCR) method in the isolates displaying reduced sensitivity against meropenem $[11,12]$.

\section{CarbaNP test}

The CarbaNP test was employed with slight changes in the original protocol to detect the carbapenemase activity [13]. After $24 \mathrm{~h}$ of incubation in the Mueller-Hinton agar, a $10-\mu \mathrm{L}$ loop full of isolates was suspended in $100 \mu \mathrm{L}$ of $20 \mathrm{mmol} / \mathrm{L}$ Tris-HCl lysis buffer (B-PERII, Bacterial Protein Extraction Reagent; Thermo Scientific Pierce, USA), vortexed for $1 \mathrm{~min}$ followed by incubation for $30 \mathrm{~min}$ at ambient temperature. The bacterial suspension was centrifuged at ambient temperature for $5 \mathrm{~min}$ at $10,000 \times g$. A small aliquot $(100 \mu \mathrm{L})$ of the supernatant from each isolate was put into an Eppendorf tube. A phenol red solution of $100 \mu \mathrm{L}(\mathrm{pH} 7.8)$ containing $0.1 \mathrm{mmol} /$ $\mathrm{L} \mathrm{ZnSO}_{4}$ (Fluka, Germany) was prepared in another Eppendorf tube. A solution of $6 \mathrm{mg} / \mathrm{mL}$ of imipenem/cilastatin and the phenol red solution were added into the tubes containing the isolates. The phenol red solution was prepared by mixing $2 \mathrm{~mL}$ of $0.5 \%$ (weight/volume) phenol red solution (Sigma, ABD) with $16.6 \mathrm{~mL}$ of distilled water followed by $\mathrm{pH}$ adjustment to 7.8 with $1 \mathrm{~N} \mathrm{NaOH}$. In lieu of the imipenem monohydrate used in the original protocol, a solution of $6 \mathrm{mg} / \mathrm{mL}$ of imipenem/cilastatin (Tienam, MSD) was used as reported in the modified protocol [14]. The tubes were incubated for $2 \mathrm{~h}$ at $37^{\circ} \mathrm{C}$ and evaluated every $15 \mathrm{~min}$. The change in the color from red to yellow was interpreted as the positive result. The test was repeated three times for the isolates where the resistance genotype was detected with the PCR method, but the test result was found to be negative. 
CIM test

A loop full of colony was picked up from the sheep blood agar plate and suspended in sterilized water. This was followed by the addition of $10 \mu \mathrm{g}$ of meropenem disk (Oxoid Ltd., Basingstoke, United Kingdom) to the bacterial suspension and incubation at $35 \pm 2{ }^{\circ} \mathrm{C}$ for $2 \mathrm{~h}$. At the end of the incubation, meropenem disk was placed on the plate on which $0.5 \mathrm{McFarland}$ standard Escherichia coli ATCC 25922 (0.5 McFarland dilution of E. coli ATCC 25922 in $5 \mathrm{~mL}$ of broth) was dispersed. The plates were incubated at $35 \pm 2{ }^{\circ} \mathrm{C}$ for at least $6 \mathrm{~h}$. At the end of the incubation period, the test was considered negative if the meropenem disk preserved its effectiveness and a zone was formed, whereas it was considered positive if a zone could not be formed around the meropenem disk because of the loss of its effectiveness caused by carbapenemase activity [9]. The test was repeated three times for the isolates in which the existence of resistance genotype had been detected with PCR method, but the test showed a negative result. K. pneumoniae ATCC BAA-1705 and K. pneumoniae ATCC 25955 strains were used as positive and negative controls, respectively.

\section{Statistical analysis}

McNemar's test was performed using SPSS for Windows (Version 17.0) software. The level of significance was defined at $p<0.05$.

\section{Results}

\section{Identification of isolates}

Out of the total CPE isolates included in the study, 77 isolates $(92.77 \%)$ were identified as $K$. pneumoniae and six isolates $(7.23 \%)$ were identified as $E$. coli. Among the isolates with no reduced sensitivity against any carbapenem, 47 isolates (94\%) were specified as K. pneumoniae, whereas three isolates were identified $(6 \%)$ as $E$. coli. It was determined that 74 isolates $(89.16 \%)$ displayed $b l a_{\mathrm{OXA}-48}$ and nine isolates $(10.84 \%)$ displayed both $b l a_{\mathrm{OXA}-48}$ and bla $_{\mathrm{NDM}-1}$ resistance genotypes.

\section{CarbaNP test results}

The CarbaNP test was found to be positive for all the isolates associated with OXA-48 and NDM-1. Among the isolates exhibiting positive result for OXA-48, 
Table I. The allocation of the CarbaNP and CIM tests' results of the isolates according to carbapenemase resistance genotypes $(n=83)$

\begin{tabular}{lccccc}
\hline Test & OXA-48 (\%) & OXA-48 and NDM-1 (\%) & Total (\%) & Sensitivity & Specificity \\
\hline CarbaNP & $60(86.96)$ & $14(100)$ & $74(89.16)$ & 89.16 & 100 \\
CIM & $67(97.10)$ & $14(100)$ & $81(97.59)$ & 97.59 & 100 \\
\hline
\end{tabular}

nine isolates (13.04\%) were determined as negative. The positivity of the CarbaNP test according to resistance genotypes is given in Table I. The test was found negative in all those isolates displaying no reduced sensitivity against any of the carbapenems.

The sensitivity of the CarbaNP test was determined as $89.16 \%$, and a specificity of $100 \%$ in the isolates studied, whereas the positive and negative predictive values of the test were detected to be 1.00 and 0.85 , respectively.

The CarbaNP test yielded a positive result in the first $15 \mathrm{~min}$ in $30(40.54 \%)$ isolates associated with OXA-48 and NDM-1 and 20 isolates producing only OXA-48. The test displayed positive results in $1 \mathrm{~h}$ in the remaining $44(59.46 \%)$ OXA-48-producing isolates.

\section{CIM test results}

The CIM test was determined to be positive in all the isolates associated with OXA-48 and NDM-1. It was observed that two of the isolates positive for OXA-48 $(2.90 \%)$ were determined as false positive. The positivity of the CIM test according to resistance genotypes is given in Table I. All the isolates were detected to be positive by the end of $6 \mathrm{~h}$. The negative isolates were incubated overnight, but the result did not change. All the isolates displaying no reduced sensitivity against any of the carbapenems were found to be negative by the CIM test.

The sensitivity of the CIM test in the isolates studied was determined to be $97.59 \%$, and the specificity was found to be $100 \%$. The positive and negative predictive values of the test were detected as 1.00 and 0.96 , respectively. We observed that the CIM test has a slightly higher sensitivity than the CarbaNP test ( $97.59 \%$ vs. $89.16 \%$, respectively; $p=0.016)$.

\section{Discussion}

Rapid detection of CPE is important both for taking effective precautions against the infection and for starting the appropriate treatment procedure [15]. Although molecular methods are considered to be the golden standard, 
laboratories prefer to apply rapid, cost-effective, and easy-to-handle tests. Furthermore, methods based on molecular approach are expensive, and the infrastructure of every laboratory is not appropriate for these tests [9]. The sensitivity and specificity of the phenotypic tests may differ depending on the enzyme type, bacterial species, and expression level of the gene that codes for the enzyme or carbapenem resistance, which may also arise from some noncarbapenemase-producing isolates [15].

Various types of CPE have spread rapidly in Turkey. Although the oxacillinases are endemic, other types of carbapenemase have also started to appear [16]. In this study, we determined the performance of CarbaNP and CIM phenotypic tests in the isolates carrying the OXA-48 enzyme, commonly observed in Turkey.

CarbaNP test is a simple and rapid test. However, the high cost of imipenem sodium is a disadvantage for the test. The drugs, imipenem and cilastatin, are inexpensive and do not cause variations in the test performance [17]. For this purpose, we employed the modified protocol of the CarbaNP test in the study [14].

On the one hand, the sensitivity of the CarbaNP test was determined to be $89.16 \%$ and its specificity to be $100 \%$. All false negative results $(13.04 \%)$ were detected in the isolates producing OXA-48 in the study. The test gave a positive result in the first $15 \mathrm{~min}$ in the isolates associated with $b l a_{\mathrm{OXA}-48}$ and $b l a_{\mathrm{NDM}-1}$ genes, whereas the test gave a positive result in $1 \mathrm{~h}$ in $59.46 \%$ of the isolates carrying bla $_{\text {OXA-48 }}$ gene. The specificity was determined to be $100 \%$ in similar studies conducted with CarbaNP test; the sensitivity value of the test ranged from $72.5 \%$ to $100 \%[8,13,18-20]$. It is reported that the sensitivity of the CarbaNP test is low, especially in the isolates carrying the $b l a_{\text {OXA-48 }}$ gene [20, 21]. Moreover, it is stated that the test gives a positive result later in the isolates carrying $b l a_{\mathrm{OXA}-48}$ gene, particularly compared with the isolates containing $\mathrm{KPC}[13,20]$. Both the low sensitivity level in the isolates and the detection of a positive result later are in compliance with this study. We believe that a slightly lower sensitivity rate found in this study compared with previous studies is due to the fact that only the isolates producing OXA-48 were included in the study.

On the other hand, the sensitivity of the CIM test was detected to be higher than the CarbaNP test $(97.10 \%)$, whereas the specificity was found to be the same $(100 \%)$. The false negative result was detected in the isolates containing OXA-48 $(2.90 \%)$ in the CIM test as well as in the CarbaNP $(13.04 \%)$ test. Studies on the evaluation of the performance of the CIM test in carbapenem-resistant Enterobacteriaceae species report that the sensitivity ranges from $98.8 \%$ to $100 \%$ and the specificity is $100 \%[9,22,23]$. Although the overnight incubation in the CIM test is specified as the greatest disadvantage of the test, Bayramoğlu et al. [23] reported that the test could be concluded in $6 \mathrm{~h}$. We also detected that all positive results were obtained in $6 \mathrm{~h}$. 
It is stated that the CarbaNP test gives false negative results, especially in the mucoid colonies containing OXA-48 and NDM-1, whereas the CIM test may be confidently used in the clinical laboratories in spite of the need for a longer incubation time [24].

Based on our investigation CarbaNP and CIM phenotypic tests are useful tools in laboratories for the identification of carbapenemase producers, especially in the regions where OXA-48 is commonly observed. However, carbapenem resistance genes need to be detected with the molecular methods in the suspicious isolates with negative CarbaNP test result.

\section{Conflict of Interest}

The authors declare no conflict of interest.

\section{References}

1. Patel, G., Bonomo, R. A.: Status report on carbapenemases: Challenge sand prospects. Expert Rev Anti Infect Ther 9, 555-570 (2011).

2. Nordmann, P., Naas, T., Poirel, L.: Global spread of carbapenemase producing Enterobacteriaceae. Emerg Infect Dis 17, 1791-1798 (2011).

3. Somily, A. M., Garaween, G. A., Abukhalid, N., Absar, M. M., Senok, A. C.: Comparison of molecular and phenotypic methods for the detection and characterization of carbapenem resistant Enterobacteriaceae. Acta Microbiol Immunol Hung 63, 69-81 (2016).

4. Karabay, O., Altindis, M., Koroglu, M., Karatuna, O., Aydemir, Ö. A., Erdem, A. F.: The carbapenem-resistant Enterobacteriaceae threat is growing: NDM-1 epidemic at a training hospital in Turkey. Ann Clin Microbiol Antimicrob 15, 16-22 (2016).

5. Hrabák, J., Chudáčková, E., Papagiannitsis, C. C.: Detection of carbapenemases in Enterobacteriaceae: A challenge for diagnostic microbiological laboratories. Clin Microbiol Infect 20, 839-853 (2014).

6. Nordmann, P.: Carbapenemase-producing Enterobacteriaceae: Overview of a major public health challenge. Med Mal Infect 44, 51-56 (2014).

7. Dallenne, C., Da Costa, A., Decre, D., Favier, C., Arlet, G.: Development of a set of multiplex PCR assays for the detection of genes encoding important beta-lactamases in Enterobacteriaceae. J Antimicrob Chemother 65, 490-495 (2010).

8. Vasoo, S., Cunningham, S. A., Kohner, P. C., Simner, P. J., Mandrekar, J. N., Lolans, K., Hayden, M. K., Patel, R.: Comparison of a novel, rapid chromogenic biochemical assay, the Carba NP test, with the modified Hodge test for detection of carbapenemase-producing Gram-negative bacilli. J Clin Microbiol 51, 3097-3101 (2013).

9. van der Zwaluw, K., de Haan, A., Pluister, G. N., Bootsma, H. J., de Neeling, A. J., Schouls, L. M.: The carbapenem inactivation method (CIM), a simple and low cost alternative for the Carba NP test to assess phenotypic carbapenemase activity in Gram negative rods. PLoS One 10, e0123690 (2015). 
10. European Committee on Antimicrobial Susceptibility Testing: Breakpoints table for interpretation of MIC's and zone diameters. Version 2.0 (2016). Available at: http://www.eucast. org/fileadmin/src/media/PDFs/EUCAST_files/Breakpoint_tables/v_6.0_Breakpoint_table. pdf. Accessed 23 December 2016

11. Poirel, L., Dortet, L., Bernabeu, S., Nordmann, P.: Genetic features of bla $a_{\mathrm{NDM}-1}$-positive Enterobacteriaceae. Antimicrob Agents Chemother 55, 5403-5407 (2011).

12. Poirel, L., Heritier, C., Tolün, V., Nordmann, P.: Emergence of oxacillinase-mediated resistance to imipenem in Klebsiella pneumoniae. Antimicrob Agents Chemother 48, 15-22 (2004).

13. Nordmann, P., Poirel, L., Dortet, L.: Rapid detection of carbapenemase-producing Enterobacteriaceae. Emerg Infect Dis 18, 1503-1507 (2012).

14. Osterblad, M., Hakanen, A. J., Jalava, J.: Evaluation of the Carba NP test for carbapenemase detection. Antimicrob Agents Chemother 58, 7553-7556 (2014).

15. Cohen Stuart, J., Leverstein-Van Hall, M. A., Dutch Working Party on the Detection of Highly Resistant Microorganisms: Guideline for phenotypic screening and confirmation of carbapenemases in Enterobacteriaceae. Int J Antimicrob Agents 36, 205-210 (2010).

16. Girmenia, C., Serrao, A., Canichella, M.: Epidemiology of carbapenem resistant Klebsiella pneumonia infections in mediterranean countries. Mediterr J Hematol Infect Dis 8, e2016032 (2016).

17. Hartl, R., Widhalm, S., Kerschner, H., Apfalter, P., Gattringer, R.: Cilastatin does not affect CarbaNP test performance for detection of carbapenemase production in Enterobacteriaceae. Wien Klin Wochenschr 129, 29-32 (2016).

18. Yusuf, E., Van Der Meeren, S., Schallier, A., Piérard, D.: Comparison of the Carba NP test with the Rapid CARB screen kit for the detection of carbapenemase-producing Enterobacteriaceae and Pseudomonas aeruginosa. Eur J Clin Microbiol Infect Dis 33, 2237-2240 (2014).

19. Dortet, L., Bréchard, L., Cuzon, G., Poirel, L., Nordmann, P.: Strategy for rapid detection of carbapenemase producing Enterobacteriaceae. Antimicrob Agents Chemother 58, 2441-2445 (2014).

20. Bayramoğlu, G., Uluçam, G., Gençoğlu Özgür, Ç., Kılıç, A. O., Aydın, F.: Comparison of the modified Hodge test and the Carba NP test for detection of carbapenemases in Enterobacteriaceae isolates. Mikrobiyol Bul 50, 1-10 (2016).

21. Tijet, N., Boyd, D., Patel, S. N., Mulvey, M. R., Melano, R. G.: Evaluation of the Carba NP test for rapid detection of carbapenemase-producing Enterobacteriaceae and Pseudomonas aeruginosa. Antimicrob Agents Chemother 57, 4578-4580 (2013).

22. Tijet, N., Patel, S. N., Melano, R. G.: Detection of carbapenemase activity in Enterobacteriaceae: Comparison of the carbapenem inactivation method versus the Carba NP test. J Antimicrob Chemother 71, 274-276 (2016).

23. Bayramoğlu, G., Uluçam, G., Gençoğlu Özgür, Ç.: Evaluation of carbapenem inactivation method for the identification of carbapenemase-producing Enterobacteriaceae strains. Mikrobiyol Bul 50, 505-507 (2016).

24. Yamada, K., Kashiwa, M., Arai, K., Nagano, N., Saito, R.: Comparison of the ModifiedHodge test, Carba NP test, and carbapenem inactivation method as screening methods for carbapenemase-producing Enterobacteriaceae. J Microbiol Methods 128, 48-51 (2016). 